\title{
STRESS a SN survey at ESO
}

\author{
Maria Teresa Botticella*t \\ INAF-Osservatorio Astronomico di Collurania Teramo \\ E-mail: botticel@na.astro.it
}

We performed the Southern inTermediate Redshift ESO Supernova Search (STRESS), a survey specifically designed to measure the rate of both SNe Ia and CC SNe, in order to obtain a direct comparison of the high redshift and local rates and to investigate the dependence of the rates on specific galaxy properties, most notably their colour. We found that the type Ia SN rate, at mean redshift $z=0.3$, is $0.22_{-0.08-0.14}^{+0.10+0.16} h_{70}^{2} \mathrm{SNu}$, while the CC SN rate, at $z=0.21$, is $0.82_{-0.24-0.26}^{+0.31+0.30} h_{70}^{2}$ $\mathrm{SNu}$. The quoted errors are the statistical and systematic uncertainties. With respect to the local value, the CC SN rate at $z=0.2$ is higher by a factor of $\sim 2$, whereas the type Ia SN rate remains almost constant. We also measured the SN rates in the red and blue galaxies and found that the SN Ia rate seems to be constant in galaxies of different colour, whereas the CC SN rate seems to peak in blue galaxies, as in the local Universe. Finally we exploited the link between SFH and SN rates to predict the evolutionary behaviour of the $\mathrm{SN}$ rates and compare it with the path indicated by observations.

Supernovae: lights in the darkness (XXIII Trobades Científiques de la Mediterrània) October 3-5 2007

Mao, Menorca, Spain

* Speaker.

${ }^{\dagger}$ Based on observations collected at the European Southern Observatory, using the $2.2 \mathrm{~m} \mathrm{MPG/ESO}$ telescope on the La Silla (ESO Programmes 62.H-0833, 63.H-0322, 64.H-0390, 67.D-0422, 68.D-0273, 69.D-0453, 72.D-0670, 72.D-0745, 73.D-0670, 74.A-9008, 75.D-0662) and using Very Large Telescope on the Cerro Paranal (ESO Programme 74.D-0714). 


\section{STRESS}

The SN rate as a function of the cosmic time is linked to some of the basic ingredients of the galaxy evolution such as mass, SFH, metallicity and environment. The rate of $\mathrm{CC} \mathrm{SNe,} \mathrm{including}$ type II and type Ib/c, gives, for an assumed initial mass function (IMF), a direct measurement of the on-going SFR because of the short evolutionary lifetimes of their progenitors. On the other hand the rate of SNe Ia echoes the long term SFH because these SNe, originating from low mass stars in binary systems, are characterized by a wide range of delay times between progenitor formation and explosion. Conversely, if a SFH is assumed, the progenitor scenarios for Ia and CC SNe can be constrained measuring their rate as a function of galaxy type and redshift.

Despite promising prospects, measurements of SN rates were very scanty so far. Most past and current high-redshift SN searches were/are aimed at detecting SNe Ia for measuring cosmological parameters, while the measurement of SN rates is a secondary goal. As such, the strategy of these searches are affected by several shortcomings [29, 23, 33, 10, 21]. In particular, these SN searches are optimized for the detection of un-reddened SNe Ia near maximum light, so that all variable sources unlikely to fall into this category are assigned a lower priority for follow-up, when not completely neglected. This strategy introduces a severe bias in the SN sample; also, in general, it prevents a reliable estimate of the rate of CC SNe.

Unlike most of the high redshift SN searches, STRESS was specifically designed to estimate the rate for both type Ia and CC SNe reducing as much as possible the biases that affect the different $\mathrm{SN}$ types. To measure the $\mathrm{SN}$ rate we counted the events observed in a selected galaxy sample. This is the same approach used to derive the local rates, and offers two advantages. By preserving the link between the observed SNe and the monitored galaxies we can investigate the dependence of the rates on galaxy colours, which depend primarily on the galaxy SFH, and on metallicity and dust extinction. In addition, the SN rates measured per unit of galaxy $B$ luminosity, that is in $\mathrm{SN}$ unit $\left(\mathrm{SNu}^{1}\right)$, can be directly compared with the local ones ${ }^{2}$.

STRESS was carried out using the Wide Field Imager (WFI) at the $2.2 \mathrm{~m} \mathrm{MPG/ESO} \mathrm{telescope}$ at La Silla, Chile. WFI is a mosaic camera consisting of $2 \times 4$ CCDs, each of $2048 \times 4096$ pixels, with a pixel scale of 0.238 arcsec and a field of view of $34 \times 33 \mathrm{arcmin}^{2}$.

The observing programme, distributed over a period of 6 years, from 1999 to 2005, can be divided into three phases. During the first year we carried out a pilot programme aimed at tuning the observing strategy and testing our software (at the beginning of our programme not all filters were available). In a second phase (2001-2003, 12 observing runs) we performed the SN search in the $V$ band targeting events at redshift $z \sim 0.2-0.3$. In the last phase (2004-2005, 4 observing runs), we performed a search in $R$ band to detect $\mathrm{SNe}$ at higher redshifts. A total of 34 nights were allocated to our programme out of which 9 were lost due to bad weather. Seeing was in the range $0.7-1.5$ arcsec, with an average around 1 arcsec.The temporal sampling, on average one observation every 3-4 months, was tuned to maximize event statistics.

Typically, in each run we observed the same fields for two consecutive nights, in the first one with the search filter, $V$ or $R$, and in the second one with a different filter to obtain colour

\footnotetext{
${ }^{1} S N u=S N(100 \mathrm{yr})^{-1}\left(10^{10} \mathrm{~L}_{\odot}^{\mathrm{B}}\right)^{-1}$

${ }^{2} \mathrm{We}$ adopt the cosmological parameters $H_{0}{ }^{3}=70 \mathrm{~km} \mathrm{~s}^{-1} \mathrm{Mpc}^{-1}, \Omega_{M}=0.3, \Omega_{\Lambda}=0.7$. Magnitudes are in the Vega System.
} 
information both for SN candidates and galaxies. Each observation, typically of 45 min integration time, was split in a sequence of three exposures jittered by 5-10 arcsec to allow a better removal of cosmetic defects, cosmic rays, satellite tracks and fast moving objects.

In order to secure the classification of the SN candidates, spectroscopic observations were scheduled about one week after the search run. In the first year, spectra were taken at the ESO3.6m telescope equipped with ESO Faint Object Spectrograph and Camera (EFOSC); in the following years, we used the FOcal Reducer and low dispersion Spectrograph (FORS1/2) at the VLT. In total 2.5 nights were allocated at the ESO3.6m telescope and 12 nights at the VLT, 4 of which were lost due to bad weather conditions. We could obtain direct spectroscopic observation only for a fraction ( $\sim 40 \%)$ of the SN candidates. In order to obtain the redshifts of the host galaxies of the remaining SN candidates, and to check for signs of the presence of Active Galactic Nucleus (AGN), we carried out a follow-up program using FORS2. The observing strategy, tuned to make the best use of non-optimal seeing and sky transparency conditions, proved to be very successful: we obtained 44 spectra of SN candidate host galaxies in a single ESO period. In addition, a dedicated service program using WFI (12 hours) was designed to complete the photometric band coverage of the fields monitored during $\mathrm{SN}$ search. We were able to secure $B, V, R, I$ imaging for 11 fields and $B, V, R$ for 5 fields.

\section{The selection of the galaxy sample and its characterization}

For the selection and the characterization of the galaxy sample, we used multi-band observations and the photometric redshift technique (photo-z), which allow us to derive the redshift, the absolute luminosity, and the rest frame colours for all the galaxies in our sample.

First we selected all the images with the best seeing and sky transparency for each field and band. Then we selected, for each band, a seeing range of $<0.15$ arcsec with the requirement that it contains the maximum number of images which hereafter are co-added. To avoid colour offsets, the co-added images in each band were convolved with an appropriate Gaussian kernel to match the PSF of the co-added image with the worst seeing.

For source detection and photometry we used SExtractor in dual image mode: source detection and classification was obtained from the original $V$ band images, since this is the band with the best $S / N$ ratio, while the magnitudes of the sources were measured on the convolved images using the same adaptive aperture as in the original $V$ images. This procedure assures that the fluxes are measured in the same physical region of each source in all bands. To separate stars from galaxies we used the SExtractor neural-network classifier fed with isophotal areas and the peak intensity of the source. The classifier returns a parameter named class_star with a value between 1 (for a point-like object) and 0 (for an extended profile).We defined as galaxies all sources having class_star $<0.9$. In principle this choice may result in an overestimate of the number of galaxies at faint magnitudes but ensures that our sample is not biased against compact galaxies.

In order to estimate the galaxy redshifts we used the spectral energy distribution (SED) template fitting technique based on the fit of the overall shape of spectra and on the detection of strong spectral features, in particular, the 4000, the Balmer or Lyman continuum breaks. Obviously the accuracy of this technique improves with the spectral range coverage: with $B$ to $R$ band photometry reliable redshifts can be obtained only in the range $0.2<z<0.8$, which may be extended 
to $z \sim 1$ by adding the $I$ band photometry. Redshifts for both very nearby galaxies, which need the $U$ band photometry, and more distant galaxies, which require infrared (IR) filter coverage, are definitely more uncertain. We set a cut-off limit for the galaxy catalogue at an apparent magnitude of $R=21.8$ mag in order to reduce the contamination from distant galaxies, which may be erroneously placed at low or intermediate redshift. This corresponds to $M_{B}^{*}$ at redshift $z=0.8$, where $M_{B}^{*}$ is a parameter of the Schechter function which fits the galaxy luminosity function [34]. We stress that for computating SN rates, we do not need the galaxy catalogue to be complete within a given volume. Nevertheless we note that at redshift $z \sim 0.2-0.3$, where the observed SN distribution peaks, the contribution to the total luminosity from galaxies fainter than $R=21.8$ mag is only $25 \%$. Photometric redshifts for the selected galaxies were computed using the code hyper- $z^{4}$ running in the predefined redshift range $0<z<0.8$. As spectral templates we tested both the observed spectra of Coleman, Wu \& Weedman (CWW) as well as the synthetic models based on Bruzual $\&$ Charlot (BC) library. Along with the redshift, the code provides the rest frame $B$-band absolute magnitude for the best fitting template. To further reduce the contamination from outliers we set a prior on the galaxy absolute luminosity. When $M_{B}$ is out of the range $(-23,-16)$ mag we examine the second solution provided by hyper- $z$, and if this is also inconsistent with the luminosity prior, we remove the galaxy from the catalog. This happened to $16 \%$ of the galaxies in the sample. Our final galaxy sample contains 43283 objects.

The accuracy of the photometric redshifts is estimated by comparing them to spectroscopic redshifts, when available, and to the photometric redshifts obtained by the COMBO-17 survey, which has a better SED sampling and used a different photo- $z$ code [34]. We quantify the reliability of the photometric redshifts by measuring the fractional error, $\Delta z=\left(z_{\mathrm{ph}}-z_{\mathrm{sp}}\right) /\left(1+z_{\mathrm{sp}}\right)$, where $z_{\mathrm{ph}}$ and $z_{\mathrm{sp}}$ are the photometric and spectroscopic redshifts, respectively. The distribution of the fractional error, both in the case of CWW template and in that of BC templates, does not show systematic off-sets: for both cases a $\langle\Delta z\rangle=0.01$ and $\sigma(\Delta) \sim 0.12$ appear consistent with our limited SED sampling. This accuracy is sufficient for our statistical analysis. In addition, the fraction of "catastrophic" outliers, galaxies with $\Delta z \geq 3 \sigma$, is relatively low, $\sim 3 \%$.

\section{The detection and classification of $\mathrm{SN}$ candidates}

Variable sources are detected as positive residuals in the difference image obtained subtracting from the search image a suitable archive image (template) acquired at a different epoch. We used SExtractor with a source detection threshold of $3 \sigma$ above the background noise and a minimum of 10 connected pixels above the threshold. In the catalogue obtained from the difference image false detections, due to imperfect removal of bright stars, cosmic rays, hot or dead pixels, outnumber real variable sources by a factor of $\sim 100$. In addition, besides the SN candidates, the detection list contains other variable sources such as fast moving objects, variable stars and variable AGNs. To clean up the detection list we used a custom-made ranking program which assigns a score to each source based on several parameters measured by SExtractor in the search, template and difference images: the class_star, the full width at half maximum (FWHM), the distance of the residual with respect to the center of the associate galaxy (if any), and apparent magnitudes of the residual

\footnotetext{
${ }^{4}$ www.web.ast.obs-mip.fr
} 
measured with different prescriptions. The scoring algorithm, tuned through extensive artificial star experiments, produces a sorted detection list. The final selection of trusted variable sources, about ten per image, was performed through visual inspection by an experienced observer. We labeled all variable sources closer than 0.5 arcsec to the host galaxy nucleus as SNAGN candidates and maintained them in the follow-up target list to reduce biases in the SN candidate selection as much as possible and classified as SN candidates those variables with a stellar profile in the difference image, irrespective of whether they appeared projected on a (host) galaxy in the search and in the template images, or not. However, in the latter case we excluded the candidate from our analysis, since we concentrate on $\mathrm{SNe}$ occurring in the galaxies within our specific sample. Spectroscopic observations were planned for all SN and SNAGN candidates but a higher priority was given to candidates flagged as SN.

To deal effectively with the large number of epochs and candidates, we developed a $M y S Q L^{5}$ database with a web interface to easily access the information remotely (e.g. during observing runs). At the end of our $\mathrm{SN}$ search programme we reviewed all variable sources recorded in our database to obtain a final classification based on all information gathered during our observing programme. A search engine allows us to identify multiple detections of the same source in different epochs and filters. This was used for the identification of AGNs which in general show long term, irregular variability. Indeed, the relatively long duration of our search makes this approach fairly efficient for AGN removal.

We could obtain direct spectroscopic observations only for 38 candidates ( $\sim 40 \%$ of the total): 7 turned out to be AGN whereas 31 were confirmed to be SNe. $25 \mathrm{SNe}(9 \mathrm{SNe} \mathrm{Ia}$ and $16 \mathrm{SNe} \mathrm{CC})$ have been observed in our galaxy sample whereas $6 \mathrm{SNe}$ discovered in galaxies with $R>21.8$ mag were excluded from the subsequent analysis. The $\mathrm{SN}$ redshifts range from $z=0.056$ to $z=0.61$. We carried out a complementary observing programme to estimate redshift and spectral type of SN host galaxies and to better constrain contamination of AGNs in the SNAGN candidate sample. We found that, out of 42, 22 host galaxies showed an active nucleus. All the candidates occurring in these galaxies were removed from the candidate list. For all these candidates the redshift of the host galaxy was estimated using the photo- $z$ technique. To avoid a possible contamination by the $\mathrm{SN}$ candidate in measuring the host galaxy colours we produced deep images, stacking only those frames obtained 6-12 months earlier than the epoch of discovery of the candidate.

In summary, the analysis presented here is based on 86 objects: 25 spectroscopically confirmed SNe, 33 SN candidates and 28 SNAGN candidates. Combining information from the long term variability history, direct and host galaxy spectroscopy we found that $\sim 50 \%$ of the variable sources originally classified as SNAGN were actually AGN. Hereafter, in the analysis of SN statistics, we assigned a weight of 0.5 to all SNAGN candidates with no spectroscopic observations. The unclassified SN and SNAGN candidates were distributed among type Ia and CC based on the observed fractions in each redshift bin for the SN subsample with spectroscopic confirmation. It turns out that, with respect to the total number, SNe Ia are $27 \pm 18 \%, 47 \pm 21 \%$ and $63 \pm 36 \%$ at redshift $z=0.1,0.3,0.5$ respectively.

\footnotetext{
${ }^{5} \mathrm{MySQL}$ is an open source database released under the GNU General Public License (GPL), http://www. mysql.com/.
} 


\section{The estimation of the control time of the galaxy sample}

The time interval during which a SN occurring in a given galaxy can be detected (the control time) depends mainly on the SN detection efficiency, the SN light curve and the amount of dust extinction. For a given $\mathrm{SN}$ type and filter $F$, the control time of the $i$-observation of the $j$-galaxy was computed as:

$$
C T_{j, i}^{\mathrm{SN}, \mathrm{F}}=\int \tau_{j}^{\mathrm{SN}, \mathrm{F}}(m) \varepsilon_{i}^{\mathrm{F}}(m) \mathrm{d} m
$$

where $\tau_{j}^{\mathrm{SN}, \mathrm{F}}(m)$ is the time spent by the $\mathrm{SN}$ in the magnitude range $m$ and $m+\mathrm{d} m$ and $\varepsilon_{i}^{\mathrm{F}}(m)$ is the detection efficiency. The total control time $C T_{j}^{\mathrm{SN}, \mathrm{F}}$ of the $j$-galaxy was computed by summing the contribution of individual observations.

The observed light curve in the band $F$ of a given SN type at redshift $z$ is given by:

$$
\begin{aligned}
m_{F}^{\mathrm{SN}}(t, z)= & M_{B}^{\mathrm{SN}}(0)+\Delta M_{B}^{\mathrm{SN}}\left(t^{\prime}\right)+K_{B F}^{\mathrm{SN}}\left(z, t^{\prime}\right) \\
& +A_{F}^{G}+A_{B}^{h}+\mu(z)
\end{aligned}
$$

where:

- $M_{B}^{\mathrm{SN}}(0)$ is the $\mathrm{SN}$ absolute magnitude at maximum in the $B$ band,

- $\Delta M_{B}^{\mathrm{SN}}\left(t^{\prime}\right)$ describes the light curve relative to the maximum,

- $K_{B F}^{\mathrm{SN}}\left(z, t^{\prime}\right)$ is the $\mathrm{K}$-correction from the $B$ to the $F$ band, $(V$ or $R)$,

- $A_{F}^{G}$ and $A_{B}^{h}$ are the Galactic and host galaxy absorption respectively,

- $\mu(z)$ is the distance modulus for the redshift $z$ in the adopted cosmology.

The light curve is translated into the observer frame by applying the time dilution effect to the rest frame SN phase $t^{\prime}=t /(1+z)$. Four basic SN types were considered: Ia, IIL, IIP, Ib/c. We adopted the same templates for the SN $B$-band light curves as in [5] and account for the observed dispersion of $\mathrm{SN}$ absolute magnitudes at maximum light by assuming a normal distribution with the mean $M_{B, 0}$ and $\sigma$ reported by [25]. We derived the $K_{B V}^{\mathrm{SN}}$ and $K_{B R}^{\mathrm{SN}}$ corrections for each SN type as a function of light curve phase with a step of 0.05 in redshift.

To estimate the host galaxy extinction we resorted to a statistical approach, based on the modelling of SN and extinction distribution in galaxies. In short, following the method described in [27], we performed a number of Monte Carlo simulations where artificial SNe were generated with a predefined spatial distribution function and were seen from uniformly distributed lines of sight. Integrating the dust column density along the line of sight for each SN we derived the total optical depth and the appropriate extinction was applied to the SN template spectrum. Repeating a number of simulations we obtained the expected distribution of SN absorptions.

We convolved the distribution of the absolute magnitude at maximum $\left(M_{B}^{\mathrm{SN}}(0)\right)$ and of the absorption due to host galaxy extinction $\left(A_{B}^{h}\right)$, so as to determine the distribution of the quantity $M_{B}^{\mathrm{SN}}(0)+A_{B}^{h}$ appearing in Eq. 4.2 , and computed $\tau_{j}^{\mathrm{SN}, \mathrm{F}}(m)$ as a weighted average of the individual times over this combined distribution. 
The detection efficiency curve as a function of the SN candidate apparent magnitude was estimated via Monte Carlo simulations: artificial point-like sources of different magnitudes were added to an image which was then searched for variable sources using the same software as in the actual search. The detection efficiency at a given magnitude is computed as the ratio between the number of recovered and injected artificial sources. Detailed simulations for a few selected fields allowed us to probe the most relevant parameters affecting the detection efficiency: the search image characteristics (observing conditions and effective search area), the search process (choice of template image and selection criteria) and the SN position in the host galaxy. We found that the seeing has the most important impact on the detection efficiency and, for a given seeing, differences in the sky transparency do not affect the shape of the efficiency curve but only its position.

Since we merged all CC subtypes, including type Ib/c IIP and IIL, the control time for CC $\mathrm{SNe}$ is computed as follows:

$$
\overline{C T}_{j}^{\mathrm{CC}}=f_{\mathrm{Ib} / \mathrm{c}} \overline{C T}_{j}^{\mathrm{Ib} / \mathrm{c}}+f_{\mathrm{IIL}} \overline{C T}_{j}^{\mathrm{IL}}+f_{\mathrm{IIP}} \overline{C T}_{j}^{\mathrm{IIP}},
$$

where the relative fractions of the different CC subtypes is assumed to be constant with redshift and equal to that observed in the local Universe, namely $20 \%$ of Ib/c and $80 \%$ of II [6], out of which $35 \%$ are IIL and $65 \%$ are IIP events [25].

\section{The measurement of $\mathrm{SN}$ rates}

Since our SN sample spans a wide redshift range $(0.056-0.61)$, we can obtain an observational constraint on the evolution of the rate by analysing the redshift distribution of the events.

In analogy with [23], and following analysis [10,11], we adopt the following power law for the reshift dependence of the rate:

$$
r^{\mathrm{SN}, \mathrm{F}}(z)=r^{\mathrm{SN}, \mathrm{F}}(\bar{z})\left(\frac{1+z}{1+\bar{z}}\right)^{\alpha^{\mathrm{SN}, \mathrm{F}}}
$$

where $r(\bar{z})$ is the rate at the mean redshift of the search, $\bar{z}$, and $\alpha$ is the evolution index. Writing the rate evolution in terms of its value at the average redshift of the search reduces the correlation between the two free parameters in Eq. (5.1).

The reference redshift $\bar{z}$ of the search is computed as the weighted average of the galaxy redshifts with weights given by the respective control times:

$$
\bar{z}^{\mathrm{SN}, \mathrm{F}}=\frac{\sum_{j=1}^{n} z_{j} \overline{C T}_{j}^{\mathrm{SN}, \mathrm{F}}}{\sum_{j=1}^{n} \overline{C T}_{j}^{\mathrm{SN}, \mathrm{F}}} .
$$

We obtained: $\bar{z}^{\mathrm{Ia}}=0.30_{-0.14}^{+0.14}$ and $\bar{z}^{\mathrm{CC}}=0.21_{-0.09}^{+0.08}$. Clearly, the lower $\bar{z}$ for CC SNe is due to their being, on average, intrinsically fainter than SNe Ia; hence their control time at a given redshift is shorter.

For a given $\mathrm{SN}$ type and filter $\mathrm{F}$, the number of expected $\mathrm{SNe}$ is given by the expression:

$$
N^{\mathrm{SN}, \mathrm{F}}(z)=\frac{r^{\mathrm{SN}, \mathrm{F}}(z)}{(1+z)} \sum_{j=1}^{n} \overline{C T}_{j}^{\mathrm{SN}, \mathrm{F}}(z),
$$


Table 1: The parameters of the rate evolution estimated for different extinction scenarios.

\begin{tabular}{lcccc}
\hline \hline & & & & \\
SN type & $\bar{z}$ & Extinction & $r(\bar{z})\left[h_{70}^{2} \mathrm{SNu}\right]$ & $\alpha$ \\
\hline \multirow{2}{*}{ Ia } & 0.30 & none & $0.13_{-0.04}^{+0.06}$ & $2.2_{-3.8}^{+3.6}$ \\
& & standard & $0.22_{-0.08}^{+0.10}$ & $4.4_{-4.0}^{+3.6}$ \\
$\mathrm{CC}$ & 0.21 & none & $0.42_{-0.12}^{+0.14}$ & $5.4_{-3.4}^{+2.8}$ \\
& & standard & $0.82_{-0.24}^{+0.31}$ & $7.5_{-3.3}^{+2.8}$ \\
& & high & $1.66_{-0.05}^{+0.32}$ & $7.8_{-3.2}^{+2.8}$ \\
\hline
\end{tabular}

where the sum is extended over the $n$ galaxies at redshift $z$ and the factor $1+z$ corrects the rate to the rest frame.

We compared the observed SN distribution, binned in redshift, to the expected distribution performing a Maximum Likelihood Estimate to derive the best fit values of the parameters $r(\bar{z})$ and $\alpha$, independently for SNe Ia and CC SNe. Our candidates come from $V$ and $R$ search programs, with no overlap. The rates at the reference redshift based on $V$ and $R$ candidates differ by less than $20 \%$ both for type Ia and CC SNe, which is well below the statistical error. Therefore, $V$ and $R \mathrm{SN}$ candidates were combined to improve the statistics.

The assumption of a particular host galaxy extinction scenario is one of the most important sources of uncertainty for $\mathrm{SN}$ rate measurements. We tested the dependence of the results on this effect by performing the fit for no extinction, a standard $(\tau(0)=1.0)$ and a high $(\tau(0)=5.0)$ extinction scenario, the last only for CC SNe. The results are reported in Table 1. With respect to the standard case, the type Ia and CC SN rates decrese respectively by a factor of 1.7 and 2 when no extinction is adopted. The effect is smaller for the SN Ia rate because SNe Ia occur, on average, in environments with a smaller amount of dust. If a high extinction correction is adopted, the CC rate increases by a factor of 2 : this can be regarded as a solid upper limit.

Systematic uncertainties in our SN rate measurements have been investigated by considering the influence of the different sources. In particular we have analysed the important role played by the AGN contamination of the SN candidate sample and the host galaxy extinction. The major uncertainty is due to the lack of a spectroscopic classification for a large fraction of the SN candidates. For the CC SN rate the estimate of the detection efficiency and the dust extinction correction also are important sources of uncertainty. Even for our relatively small SN sample, the statistical and systematic uncertainties are comparable.

\section{Comparison with the local $\mathrm{SN}$ rates and other measurements at intermediate redshift.}

The local SN rates measured by collecting data of five photographic SN searches $[6$, and reference therein] are: $r^{I a}=0.17 \pm 0.04$ and $r^{C C}=0.41 \pm 0.17 h_{70}^{2} \mathrm{SNu}$ at $\bar{z}=0.01$. As can be seen the $\mathrm{SN}$ Ia rate in $\mathrm{SNu}$ appears constant, within the uncertainties, up to $z=0.3$, whereas the $\mathrm{SN} \mathrm{CC}$ 
Table 2: SN rates as a function of galaxy color $\left[\mathrm{SNu} h^{2}\right]$

\begin{tabular}{lcccc}
\hline \hline SN type & \multicolumn{2}{c}{$r(z=0.01)$} & \multicolumn{2}{c}{$r(z=0.25)$} \\
& red & blue & red & blue \\
\hline \multirow{2}{*}{ Ia } & $0.20 \pm 0.04$ & $0.19 \pm 0.04$ & $0.16_{-0.18}^{+0.13}$ & $0.23_{-0.09}^{+0.13}$ \\
& & & & \\
CC & $0.10 \pm 0.04$ & $0.99 \pm 0.15$ & $0.57_{-0.30}^{+0.48}$ & $1.50_{-0.48}^{+0.66}$ \\
& & & & \\
All & $0.30 \pm 0.06$ & $1.18 \pm 0.16$ & $0.73_{-0.31}^{+0.50}$ & $1.73_{-0.45}^{+0.67}$ \\
\hline
\end{tabular}

rate increases by a factor of 2 already at $z=0.21$. The different evolutionary behaviour of CC and Ia $\mathrm{SN}$ rates implies that their ratio increases by a factor of $\sim 2$ from the local Universe to a lookback time of "only" $3 \mathrm{Gyr}(z=0.25)$. Considering that, in this same redshift range, the cosmic SFR nearly doubles, the evolution with redshift of the ratio $r^{C C} / r^{I a}$ requires that a significant fraction of SN Ia progenitors has a delay time longer that $2-3 \mathrm{Gyr}$.

The interpretation of the evolution of the rate in $\mathrm{SNu}$ is not straightforward, as it reflects both the redshift dependence of both the SFH and the $B$-band luminosity. Indeed, the $B$-band luminosity, with a contribution from both old and young stars, evolves with a different slope to the on-going SFR. We acknowledge that the estimate of the SN rate evolution with redshift depends on the adopted extinction correction. For instance, the ratio between the rate at $\mathrm{z}=0.21$ and the local rates for CC SNe varies from 1.6 to 2.8, when no or high extinction correction is applied. However, the fact that the CC SN rate increases faster than the SN Ia rate appears to be a robust result.

In the local Universe, SN rates as a function of galaxy colours were derived by [6] for optical bands and by [18] for optical-infrared bands. The SN Ia rate per unit $B$ luminosity appears almost constant in galaxies with different $U-V$ color, whereas the CC SN rate strongly increases from red to blue galaxies, a trend very similar to that of the SFR. This indicates that a fraction of SN Ia progenitors has long delay times, whereas $\mathrm{CC} \mathrm{SNe}$ are related to young massive stars. Given the diagnostic power of the trend of the SN rates with the colour of the parent galaxy we investigate here this trend at intermediate redshift. We split up our galaxy sample into blue and red subsamples, according to the observed $B-V$ color. We took the rest frame color of the $S a \mathrm{CWW}$ template as a reference. The local galaxy sample of [6] was divided in the same way. $U-V$ or $B-R$ colours could be more sensitive tracers of the stellar populations, but $U$-band photometry is not available for our galaxy sample and $R$-band is not available for the local sample.

The $\mathrm{SN}$ rates in the blue and red galaxy samples were computed by distributing the unclassified SN and SNAGN candidates among type Ia and CC SNe, based on the observed fractions of the spectroscopically confirmed SNe in each of the two galaxy samples (in the red sample SNe Ia are $67 \%$, whereas they are only $25 \%$ of the blue sample).

The $\mathrm{SN}$ rates (in $\mathrm{SNu}$ ) in the local Universe and at redshift $z=0.25$ for the red and blue galaxy subsamples are listed in Table 2.

We found very similar trends in the local Universe and at redshift $z=0.25$ both for type Ia and CC SNe. It appears that while the SN Ia rate is almost costant in galaxies of different colors, the CC SN rate always peaks in blue galaxies. This result is consistent with that of [32] who, after 


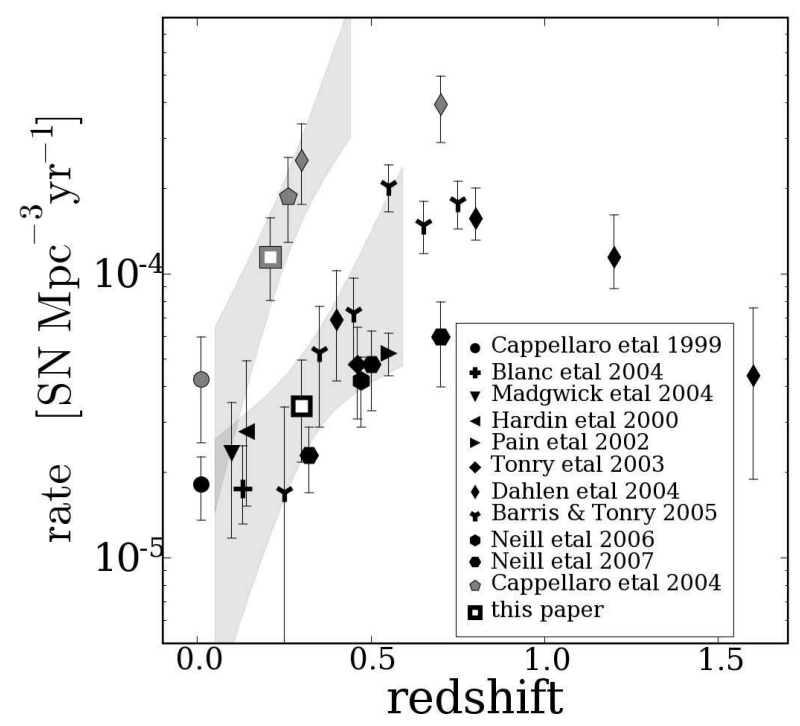

Figure 1: Observed SN rates as function of redshift from different authors as indicated in the legend. The black (gray) symbols indicate SN Ia (SN CC) rate measurements. The shaded area represents the $1 \sigma$ confidence level of our rate evolution estimate as deduced from the MLE fit.

comparison with the measurements of [18], found that the SN Ia rate as a function of galaxy colours does not evolve significantly with redshift. The rapid increase with redshift of the CC SN rate both in blue and red galaxies can be attributed to an increasing proportion of star forming galaxies in the red sample going from low to intermediate redshift.

After accounting for the evolution with redshift of the blue luminosity density $\left(j_{B}(z)=\right.$ $\left.(1.03+1.76 \times z) \quad 10^{8} L_{\odot}^{B} \mathrm{Mpc}^{-3}\right)$, we have compared our estimates with all other measurements at intermediate redshift available in the literature and found that they are consistent, within the relatively large errors. We found that the rates of the Ia and CC SN per unit of co-moving volume are: $r^{\text {Ia }}(z=0.30)=0.34_{-0.15-0.22}^{+0.16+0.21} 10^{-4} h_{70}^{3} \mathrm{yr}^{-1} \mathrm{Mpc}^{-3}$ and $r^{\mathrm{CC}}(z=0.21)=1.15_{-0.33-0.36}^{+0.43+0.42}$ $10^{-4} h_{70}^{3} \mathrm{yr}^{-1} \mathrm{Mpc}^{-3}$ where both statistical and systematic errors are indicated. Also local rates are converted into volumetric rates: $r^{\mathrm{Ia}}(z=0.01)=0.18 \pm 0.0510^{-4} h_{70}^{3} \mathrm{yr}^{-1} \mathrm{Mpc}^{-3}$ and $r^{\mathrm{CC}}(z=$ $0.01)=0.43 \pm 0.1710^{-4} h_{70}^{3} \mathrm{yr}^{-1} \mathrm{Mpc}^{-3}$. With respect to the rates in $\mathrm{SNu}$, the volumetric rates evolve more rapidly with redshift, due to the increase of the $B$ band luminosity density. We find an increase of a factor of $\sim 2$ at $z=0.3$ for SNe Ia, and a factor of $\sim 3$ at $z=0.21$ for CC SNe.

Measurements of Ia and CC SN rate as a function of redshift are shown in Fig. 1, where those originally given in $\mathrm{SNu}[13,3,7]$ were converted into measurements per unit volume as above.

As can be seen, the few measurements of the CC SN rate appear to be fully consistent, while those of the SN Ia rate show a significant dispersion which increases with redshift, in particular in the range $0.5<z<0.7$ where the values of [2] and [10] are 2-3 times higher that those of [23] and [22]. Our estimate of the SN Ia rate is consistent with all other measurements in the redshift range we explored; our result does not help to discriminate between the steep trend suggested by 
the [2] and [10] measurements and the slow evolution indicated by the [22] measurement. The robust indication from the current data appears that the $\mathrm{SN}$ Ia rate per unit volume at redshift 0.3 is a factor of $\sim 2$ higher than in the local Universe, while in the same redshift range the CC SN rate increases by a factor of $\sim 5$.

\section{Comparison with the predicted evolution of the $\mathrm{SN}$ rates}

We have exploited the link between SFH and SN rates to predict the evolutionary behaviour of the SN rates and compare it with the path indicated by observations. Given the short lifetime of their progenitors $(<30 \mathrm{Myr})$, there is a simple, direct relation between the $\mathrm{CC} \mathrm{SN}$ and the current SF rate:

$$
r^{\mathrm{CC}}(z)=K^{\mathrm{CC}} \times \psi(z)
$$

where $\psi(z)$ is the SFR and $K^{C C}$ is the number of CC SN progenitors from a $1 M_{\odot}$ stellar population:

$$
K^{\mathrm{CC}}=\frac{\int_{m_{l}^{\mathrm{CC}}}^{m_{u}^{\mathrm{CC}}} \phi(m) d m}{\int_{m_{L}}^{m_{U}} m \phi(m) d m}
$$

where $\phi(m)$ is the IMF, $m_{L}-m_{U}$ is the total stellar mass range, and $m_{l}^{\mathrm{CC}}-m_{u}^{\mathrm{CC}}$ is the mass range of CC SN progenitors.

The predicted evolution of the CC SN rate with redshift has been computed assuming the mass range 8-50 $M_{\odot}$ for CC SN progenitors and three representative SFHs: the piecewise linear fit of selected SFR measurements by [17], the fit to the SFR measurements from the $H \alpha$ emission line by [16], the prescriptions by [15]. All these SFHs were converted to the same IMF, a modified Salpeter IMF (SalA) with a turnover below $0.5 M_{\odot}$ and defined in the mass range $m_{L}=0.1 M_{\odot}$ to $m_{U}=120 M_{\odot}$ [1]. The measurements of the CC SN rate per unit volume and the predicted evolutionary behaviours are shown in Fig 2.

The comparison with the observed evolution confirms the steep increase with redshift indicated by most recent SFH estimates. Specifically, we found a good agreement with the predictions from the SFH inferred through $\mathrm{H} \alpha$ luminosity [16], while the SFHs from FIR luminosity [17] overestimate the CC SN rate, unless a higher extinction correction and/or a narrower range for the progenitor masses is adopted. This result illustrates how interesting clues can be obtained by comparing the SN CC rate to other SFR tracers, in the same galaxy sample, to verify the reliability of the techniques used to derive SFR estimates and the adequacy of the dust extinction corrections.

Following [12] the cosmic evolution of the SN Ia rate is given by:

$$
r^{\mathrm{Ia}}(t)=k_{\alpha} A^{\mathrm{Ia}} \int_{\tau_{i}}^{\min \left(t, \tau_{x}\right)} f^{\mathrm{Ia}}(\tau) \psi(t-\tau) d \tau
$$

where $k_{\alpha}$ is the number of stars per unit mass of the stellar generation, $A^{\text {Ia }}$ is the realization probability of the SN Ia scenario (the number fraction of stars from each stellar generation that end up as $\mathrm{SN} \mathrm{Ia}), f^{\mathrm{Ia}}(\tau)$ is the distribution function of the delay times and $\psi(t-\tau)$ is the star formation rate at the epoch $t-\tau$. The integration is extended over all values of the delay time $\tau$ in the range $\tau_{i}$ and $\min \left(t, \tau_{x}\right)$, with $\tau_{i}$ and $\tau_{x}$ being the minimum and maximum possible delay times for a given progenitor scenario. Here we assumed that both $k_{\alpha}$ and $A^{\text {Ia }}$ do not vary with cosmic time. 


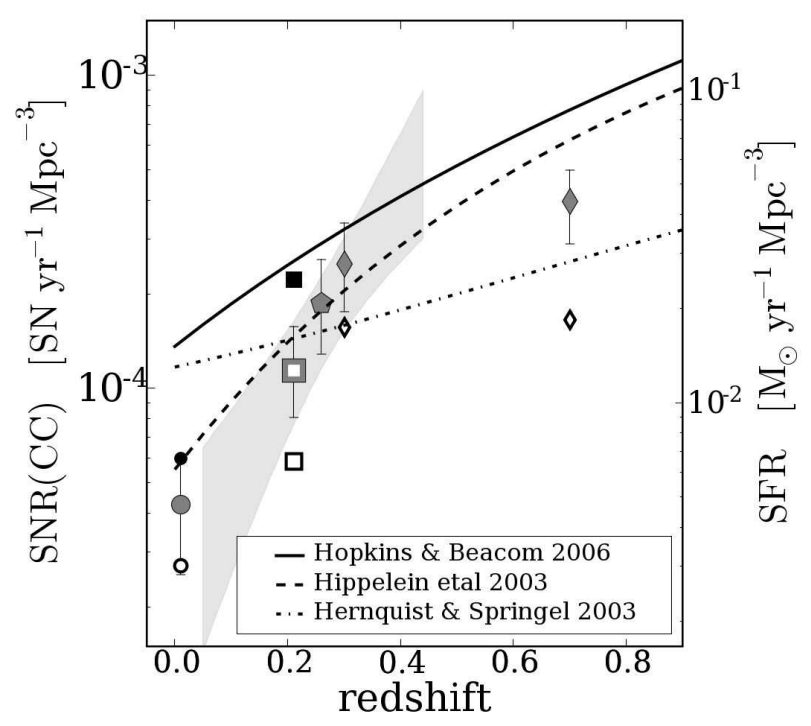

Figure 2: Comparison between the SN CC and SF rate evolution. Symbols are as in Fig. 1 with additional open symbols (measurements not corrected for extinction) and filled black symbols (estimates for the high extinction correction). Lines are selected SFR evolutions from the literature. All SFHs have been scaled to the SalpeterA IMF.

To estimate the cosmic evolution of the SN Ia rate we convolved the SFH of [17] with various formulations of DTD representative of different approaches to model the SN Ia rate evolution: three models from [12], and two different parametrizations [19,30] designed to address some specific observational constraints, regardless the correspondence to a specific progenitor scenario.

The predicted evolutionary behaviours of the SN Ia rate are compared with all published measurements in Fig. 3. In all cases, the value of $k_{\alpha} A^{\text {Ia }}$ was fixed to match the value of the local rate; depending on the model it ranges between 3.4-7.6 $\times 10^{-4}$. This normalization implies that, for the adopted SalA IMF, and assuming a mass range for the progenitors of $3-8 M_{\odot}$, the probability that a star with suitable mass becomes a SN Ia is $\sim 0.01-0.03$.

All DTDs appear to predict a SN Ia rate evolution consistent with the observations, with the exception of the "wide" DD model, which appears too flat. On the other hand none of the DTD functions, with the adopted SFH, is able to reproduce at the same time the very rapid increase from redshift 0 to 0.5 suggested by some measurements $[2,10]$ and the decline at redshift $>1$ [10]. Given the current uncertainties of both SN Ia rate and SFH it is difficult to discriminate between the different DTD functions and hence between the different SN Ia progenitor models. To improve on this point, more measurements of SN Ia rate at high redshifts are required to better trace the rate evolution. At the same time measurements in star forming and in passivly evolving galaxies over a wide redshift range can provide important evidence about the SN Ia progenitor models. 


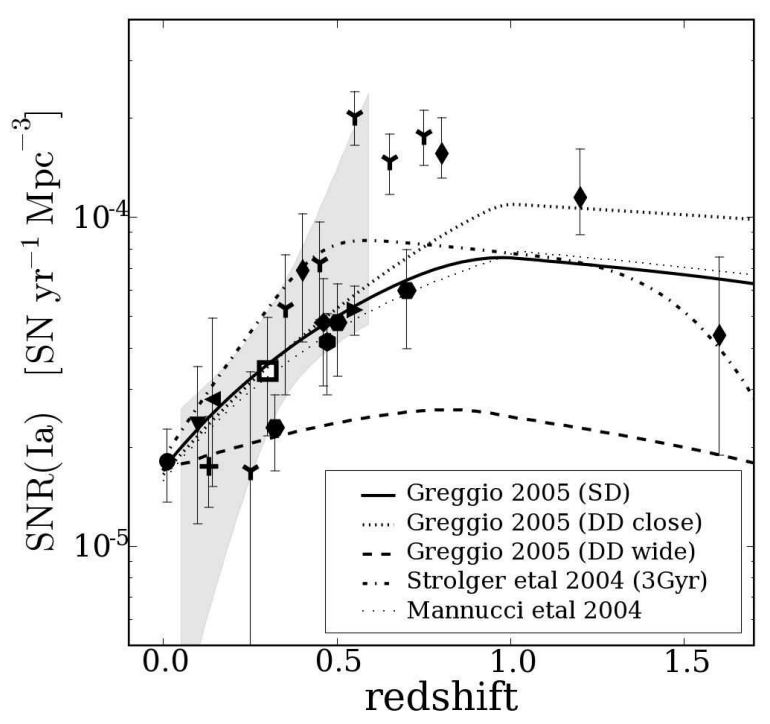

Figure 3: SN Ia rate measurements fitted with different DTD functions and the SFH by Hopkins. Symbols for measurements are as in Fig. 1.

\section{References}

[1] Baldry, I. K., \& Galzebrook, K. 2003, , 593, 258

[2] Barris, B. J., \& Tonry, J. L. 2006, , 637, 427

[3] Blanc, G., et al. 2004, , 423, 881

[4] Cappellaro, E., Turatto, M., Benetti, S., et al. 1993 , 273,383

[5] Cappellaro, E., Turatto, M., Tsvetkov, D. Y., et al. 1997, , 322, 431

[6] Cappellaro, E., Evans, R., \& Turatto, M. 1999, , 351, 459

[7] Cappellaro, E., Riello, M., Altavilla, G., et al. 2005, , 430, 83

[8] Coleman, G. D., Wu, C.-C., \& Weedman, D. W. 1980, , 43, 393

[9] Dahlén, T., \& Fransson, C. 1999, , 350, 349

[10] Dahlén, T., Strolger, L. G., Riess, A. G., et al. 2004, , 613, 189

[11] Gal-Yam, A., \& Maoz, D. 2004, , 347, 942

[12] Greggio,L., $2005,441,1055$

[13] Hardin, D., Afonso, C., Alard, C., et al. 2000, , 362, 419

[14] Hendry, M. A.,Smartt, S. J., Maund,J. R., et al. 2005, 359,906

[15] Hernquist, L., \& Springel, V. 2003, , 341, 1253

[16] Hippelein, H., Maier, C., Meisenheimer, K., et al. 2003, , 402, 65 
[17] Hopkins, A. M., \& Beacom, J. F., 2006, , 651, 142

[18] Mannucci, F., Della Valle, M., Panagia, N., et al. 2005, , 433, 807

[19] Mannucci, F., Della Valle, M., \& Panagia, N. 2006, , 370, 773

[20] Maoz, D., \& Gal-Yam, A., 2004, , 347, 951

[21] Neill, J. D., Sullivan, M., Balam, D., et al. 2006, , 132, 1126

[22] Neill, J. D., Sullivan, M., Balam, D., et al.2007, ArXiv Astrophysics e-prints, astro-ph/0701161

[23] Pain, R., Fabbro, S., Sullivan, M., et al. 2002, , 577, 120

[24] Perlmutter, S.,Aldering, G., Goldhaber, G., et al. 1999, , 517, 565

[25] Richardson, D., Branch, D., Casebeer, D., et al. 2002, , 123, 745

[26] Richardson, D., Branch, D., \& Baron, E. 2006, , 131, 2233

[27] Riello, M. \& Patat, F. 2005, MNRAS, 362, 671

[28] Riess, A. G., Filippenko, A. V., Challis, P., et al. 1998, , 116, 1009

[29] Schmidt, B. P., Suntzeff, N. B., Phillips, M. M., et al. 1998, , 507, 46

[30] Strolger, L.-G., Riess, A.G., Dahlén, T., et al. 2004 , 613,200

[31] Strolger, L.-G., \& Riess, A. G. 2006, , 131, 1629

[32] Sullivan, M., et al. 2006, , 648, 868

[33] Tonry, J. L., Schmidt, B. P., Barris, B., et al. 2003, , 594, 1

[34] Wolf, C., Meisenheimer, K., Rix, H.-W., et al. 2003, , 401, 73 